cause the nitric acid treatment can be scaled up, the availability of open nanotubes is limited only by the availability of the capped feedstock. Ebbesen and coworkers, and Hwang, have also obtained good tube-opening results with a solution of potassium permanganate in acidic solution (T. Ebbesen, K. C. Hwang, personal communication).

Selective removal of tube tips by nitric acid shows that the pentagon carbon atoms and the carbon atoms under strain at the tips are reactive but that the hexagonal network of $s p^{2}$-bonded carbon on the tube sides is not, on the timescale of treatment used in ref. 4. The ability of nitric acid to chew selectively along a line of pentagons, 'drilling' inwards through a series of corners in a nested polyhedral particle (R. S. R. and S. Subramoney, unpublished results) without destroying the hexagonal sheets, may lead to new recipes for carbon-coated nanocrystals where the nanocrystals are formed by techniques analogous to those used by Tsang et al. to fill nanotubes.

One aspect of ref. 4 deserves special scrutiny. One could infer from the procedures used that the final volume filling of $\mathrm{NiO}$ on conversion of a nickel precursor is determined by the concentration of nickel nitrate solution used. Straightforward calculation shows that a volume filling of only 0.27 per cent would result. But the lengths given as typical are $20 \mathrm{~nm}$ for $\mathrm{NiO}$ crystallites and $200 \mathrm{~nm}$ for nanotubes and the $\mathrm{NiO}$ crystallite fills the nanotube essentially completely over its 20 -nm length ${ }^{4}$. So the filling cannot simply be a question of the bulk solution concentration. The roughly 10 per cent filling with $\mathrm{NiO}$ means that the nickel nitrate precursor (a solid) must essentially completely fill the tube (taking account of molecular weights and respective densities). How does nearly 40 times 'too much' $\mathrm{NiO}$ end up, following calcination, in the nanotube, or how could the tube be filled completely with a solid precursor? I leave it to the reader to ponder the implications of precipitation of solids inside the nanotubes when the bulk solution outside them is subsaturated.

We may speculate about possibilities that filling from solution can offer. One may think of the internal volume of a nanotube as a test tube for studying chemical reactions, or the thermodynamics or kinetics of phase transitions, in small spaces. For an ionic solution, is the concentration of ions in the solution inside the tube the same as in the bulk solution, or do unusual concentration gradients develop? If one forces a non-wetting liquid ${ }^{14}$ such as mercury into a tube with high pressure, will it stay in the tube when pressure is released? If so, and the filled tube is cooled to below the superconducting transition temperature of bulk $\mathrm{Hg}$ (about $4 \mathrm{~K}$ ), perhaps interesting phenomena will be observed. At room temperature, will en- capsulated $\mathrm{Hg}$ be a liquid? For the chemist chef who desires peas-in-a-pod, can opened nanotubes be filled with $\mathrm{C}_{60}$ ?

One wonders, too, how enclosed nanocrystals whose expansion is limited radially will melt. Topological constraints may govern phase transitions; for instance, preliminary theoretical efforts show that argon will have difficulty forming a complete solid phase inside some tube geometries even though the temperature is below the bulk freezing value, because of the difficulty of growing a nonfrustrated cylindrical crystal inwards from the inner tube perimeter to the centre (M. Maddox and K. E. Gubbins, personal communication). The dynamics of water or of a long-chain polymer inside nanotubes could be fascinating and could be studied by NMR.

One wants to believe in quantum wires based on long nanotubes that have been completely filled with conducting metals, and progress in improving yield and quality of nanotubes continues at a good rate. But in my opinion this is a more distant goal than immediate studies of incorporating materials of relatively short length into opened nanotubes, and investigating their various properties. Progress in fundamental areas and in applications that do not rely on perfect filling should come first, such as studies of the thermodynamics and dynamics of solutions or solids inside nanotubes, and new catalysts.

But if we may dream of nanowires, how about taking a scanning probe microscope nanotip that is a carbon nanotube, clipping off its end by a wet chemical etch, and dipping it into an appropriate solution to produce a magnetic encapsulate such as $\mathrm{Fe}$ or $\mathrm{Ni}$ at the tip end? Perhaps such a tip could detect atom-specific magnetic resonance of biomolecules on surfaces.

Rodney S. Ruoff is in the Molecular Physics Laboratory, SRI International, 333 Ravenswood Avenue, Menlo Park, California 94025, USA.

1. lijima, S. Nature $354,56-58$ (1991)

2. Ajayan, P. M. \& lijima, S. Nature 361, 333-334 (1993)

3. Guerret-Piécourt, C., Le Bouar, Y., Loiseau, A. \& Pascard, H. Nature 372, 761-765 (1994).

Tsang, S. C., Chen, Y. K., Harris, P. J. F. \& Green, M. L. H Nature 372, 159-162 (1994).

5. Bethune, D. S. etal. Nature 363, 605-607 (1993)

6. lijima, S. \& Ichihashi, T. Nature 363, 603-605 (1993)

7. Seraphin, S. et al. Nature 362, 503 (1993).

8. Subramoney, S. et al. Carbon 32, 507-513(1994).

9. Ruoff, R. S., Lorents, D. C., Chan, B., Malhotra, R. \& Subramoney, S. Science 259, 346-348 (1993)

10. Saito, Y. Fullerenes: Recent Advances in the Chemistry and Physics of Fullerenes and Related Materials (eds Kadish. K. M. \& Ruoff, R. S.) 1419-1432 (The Kadish, K. M. \& Ruoff, R. S.) 1419-1432 (The
Electrochemical Society, Pennington, New Jersey, 1994).

11. Smalley, R. E. Mater. Sci. Engng B19, 1-7 (1993)

12. Colbert, D. T. et al. Science 266, 1218 (1994).

13. Lin, X., Wang, X. K., Chang, R. P. H., Ketterson, J. B. \& Dravid, V.P. Fullerenes: Recent Advances in the Chemistry and Physics of Fullerenes and Related Materials (eds Kadish, K. M. \& Ruoff, R. S.) 1478-1486 (The Electrochemical Society, Pennington, New Jersey, (The Electro
1994).

14. Dujardin, E., Ebbesen, T. W., Hiura, H. \& Tanigaki, K. Science 265, 1850-1852 (1994).

\section{Celestial graffiti}

LAST week Daedalus proposed the scanning electron telescope. It was an orbiting launcher for strong electron and positive-ion beams (both together, to maintain overall charge-neutrality). The beams were to be aimed at planetary targets, so that earthbound telescopes could study the luminosity of their impact. He now proposes aiming the beams down towards the Earth.

When high-energy particles hit the high atmosphere, they excite an auroral glow. The shifting, filmy, magical curtains of the polar aurora are produced by the impact of solar-wind particles on the air molecules about 150 kilometres up. The molecules luminesce as they decay back to their ground state. Aurorae can have many colours: red (the 'proton glow' from protons in the solar wind), and yellow, green and violet from various spectral bands of electron-excited nitrogen and oxygen molecules.

A powerful, narrow particle beam directed from above should create a very bright 'point-aurora' at its site of impact. The colour and intensity of the auroral glow could be controlled by varying the energy and particle density of the beam. The positive and negative beams would create point-aurorae of different colours, which could be scanned independently around the sky. The application is obvious: celestial colour television. From space, it should be possible to scan a picture over the atmosphere 100 kilometres across and 150 kilometres up, and visible over an area the size of central Europe.

In the daytime, the brightest auroral picture would be imperceptible. Celestial television will come into its own at night. Low-orbit satellites will speed across the heavens trailing huge pictures in their wake, like publicity banners behind an invisible plane. Geosynchronous satellites will paint a stable frame that will fill the same chunk of sky night after night. At first Daedalus hoped for educational programmes, with leading astronomers pointing out planets and constellations to the upgazing masses. But the dream soon faded. He now expects the sky to fill up rapidly with the usual trash, advertisements and all. One consolation is that the pictures would be silent. Aurorae make no noise of their own, and even the radio emission from particle impact would be hard to modulate into a useful soundtrack. Furthermore, the Earth's ever-changing magnetic field will sabotage the linescan, making the images wobble unsteadily. With luck this undignified wavering will discourage the more pompous nonsense, leaving the sky to the likes of Tom and Jerry. 